Review Article:

\title{
A Systematic Review on the Risk Factors of Congenital Hypothyroidism
}

Mahin Hashemipour ${ }^{1,2}$ (D), Payam Samei ${ }^{1,2^{*}}$ (D), Roya Kelishadi ${ }^{2}$ (D), Silva Hovsepian ${ }^{2,3}$ (D), Neda Hani Tabaei Zavareh ${ }^{4}$ (D)

1. Isfahan Endocrine and Metabolism Research Center, Isfahan University of Medical Sciences, Isfahan, Iran.

2. Child Growth and Development Research Center, Research Institute for Primordial Prevention of Non-communicable Disease, Isfahan University of Medical Sciences, Isfahan, Iran.

3. Imam Hossein Children's Hospital, Isfahan University of Medical Sciences, Isfahan, Iran.

4. Department of Public Health, Massachusetts College of Pharmacy and Health Sciences, Boston, Massachusetts, United States.

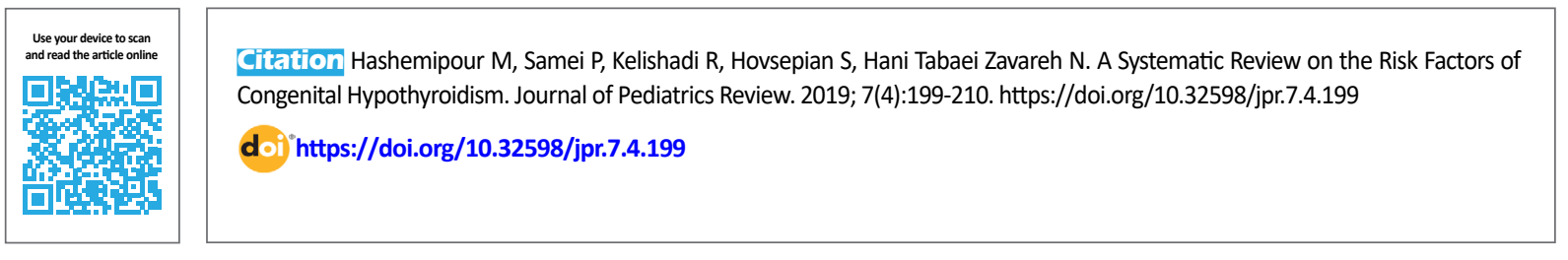

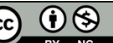

Article info:

Received: 21 July 2018

First Revision: 30 July 2018

Accepted: 03 February 2019

Published: 01 October 2019

\section{Key Words:}

Congenital hypothyroidism, Permanent, Transient, Risk factor

\section{A B STRACT}

Context: Congenital Hypothyroidism $(\mathrm{CH})$ is the most common endocrine disorder and causes of preventable mental retardation in children.

Objective: We aimed to review the reported $\mathrm{CH}$-related risk factors systematically.

Data Sources: In this review, all types of human studies on the risk factors related to the occurrence or high rate of $\mathrm{CH}$ were included. An electronic search was conducted in international and national electronic databases. The following keywords were used: "Congenital Hypothyroidism" AND "risk factor".

Study Selection: In this review, 373 papers (from PubMed: 199; Scopus: 36; ISI: 53, SID: 55, Ovid: 11; Science Direct: 19) were identified through electronic database search. A total of 98 articles were assessed for their eligibility, from which 60 qualified articles were selected for final evaluation. Most of the studies have cross-sectional, case-control, and prospective design.

Data Extraction: The current review was conducted and reported following the PRISMA (preferred reporting items for systematic reviews and meta-analyses) statement.

Results: Reported risk factors for transient $\mathrm{CH}$ were as follows; iodine deficiency and excess, prematurity, advanced maternal age, male gender, retinopathy of prematurity, twin pregnancy, maternal autoimmune thyroid disease, intrauterine growth retardation, and cesarean delivery. Reported risk factors for permanent $\mathrm{CH}$ with dysgenesis of the thyroid gland were as follows; female gender, familial history of $\mathrm{CH}_{\text {, }}$ birth in geographical areas with a high rate of the disease, advanced maternal age, ethnicity (Caucasians), but not seasonality. Reported risk factors for permanent $\mathrm{CH}$ with dyshormonogenesis were a familial history of $\mathrm{CH}$ and origin of both parents from the high-risk geographical region.

Conclusions: By using this information, we could plan more etiologic studies to investigate the pathogenesis of the disorder, design interventional studies for the known modifiable risk factors to reduce the rate of $\mathrm{CH}$ in our region. Also, for risk factors with limited evidence, more studies should be performed.

\section{* Corresponding Author:}

Payam Samei, MD.

Address: Department of Pediatrics, Schoole of Medicine, Isfahan University of Medical Sciences, Isfahan, Iran.

Tel: +98 (913) 1294269

E-mail: payam.sameii@gmail.com 


\section{Context}

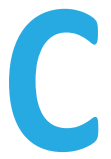

ongenital Hypothyroidism $(\mathrm{CH})$ is the most common endocrine disorder and causes of preventable mental retardation in children. It is defined as thyroid hormone deficiency at birth $(1,2)$. It is classified as primary and secondary. Primary causes include defects in thyroid gland development (thyroid dysgenesis) or deficiencies in thyroid hormone synthesis (thyroid dyshormonogenesis) (1, 2).

$\mathrm{CH}$ screening program is considered the most practical and effective method of $\mathrm{CH}$ diagnosis as the disorder has not any specific signs and symptoms at birth or during neonatal period. It is a routine practice in developed countries and many developing countries. Findings of $\mathrm{CH}$ screening from different regions and countries indicate great variability in the incidence and etiology of $\mathrm{CH}$. In accordance with $\mathrm{CH}$ screening, etiological factors, and various risk factors of the disorder have been identified and reported in previous studies $(3,4)$.

Findings of the studies demonstrate the importance of etiological studies for better understanding of the pathogenesis of $\mathrm{CH}$, as well as its related risk factors to conduct further preventative strategies. The investigation of modifiable risk factors for $\mathrm{CH}$ is important because of the potential to prevent $\mathrm{CH}$, especially in regions with a high rate of $\mathrm{CH}$.

Based on the current evidence, several individual and environmental factors affect $\mathrm{CH}$ such as gender, birth weight, race, age, consanguinity, parental education, type of labor, birth order, twin and drug usage during pregnancy (5-8). It is believed that many other risk factors might influence the occurrence of $\mathrm{CH}$ (5-12). Confirming the causality between these risk factors and $\mathrm{CH}$ and identifying them might be helpful even in decreasing the incidence of $\mathrm{CH}$. More practically, it can help have a higher index of suspicion for $\mathrm{CH}$ in neonates with the identified risk factors.

\section{Objective}

Though there are different studies in this field, the results are not conclusive enough, and it is suggested that systematically reviewing of $\mathrm{CH}$-related risk factors would provide us more appropriate information for designing our future etiological and preventative research. So we aimed to review the reported $\mathrm{CH}$-related risk factors systematically.

\section{Data Sources}

In this study, we systematically reviewed all studies which investigated $\mathrm{CH}$ risk factors. The protocol of this study was approved by the Ethics Committee of Isfahan University of Medical Sciences. An electronic search was conducted in international electronic databases, including PubMed, Cochrane, Scopus, ISI, Web of Science, Ovid, Science direct, as well as Persian databases such as IranMedex, IranDoc, and Scientific Information Database (SID). The keywords of "congenital hypothyroidism" (Mesh) AND "risk factor" (Mesh) were used in the Title and the abstract. The latest search was conducted on the 29th September 2017.

\section{Study Selection}

In this review, all types of human studies on the risk factors related to the occurrence or high rate of $\mathrm{CH}$ were included without any time limitation. The included articles were in English and Persian. The search was performed without any time limitation until September 2017. Inappropriate or repeated papers were excluded. The titles of all searched articles were reviewed and studied, and repeated items were excluded. Two researchers carefully studied the full text of selected articles and excluded irrelevant papers. A secondary search was conducted from the references of the selected papers.

\section{Data Extraction}

The Preferred Reporting Items for Systematic Reviews and Meta-Analyses (PRISMA) checklist was used for reporting systematic reviews. The quality of the documents was evaluated independently by two research experts regarding the objective of each study, methods, sample size, sampling method, data collection tool, variable evaluation status, and evaluated target group. Disagreements were resolved by consensus, mutual discussion, and consulting with an expert in the field of $\mathrm{CH}$. From each finally included article, the following information was extracted; authors, place of the study, ethnicity, year of publication, sample size, study design, and reported risk factors.

In this review, 373 papers (PubMed: 199; Scopus: 36; ISI: 53, SID: 55, Ovid: 11; Science Direct: 19) were identified through electronic database search. A total of 98 articles were assessed for eligibility, of which 60 qualified articles were selected for final evaluation (Figure 1). Details of all selected studies were presented in Table 1 (5, 8, 9, 12-67). 
Articles identified through electronic database search $(n=373)$

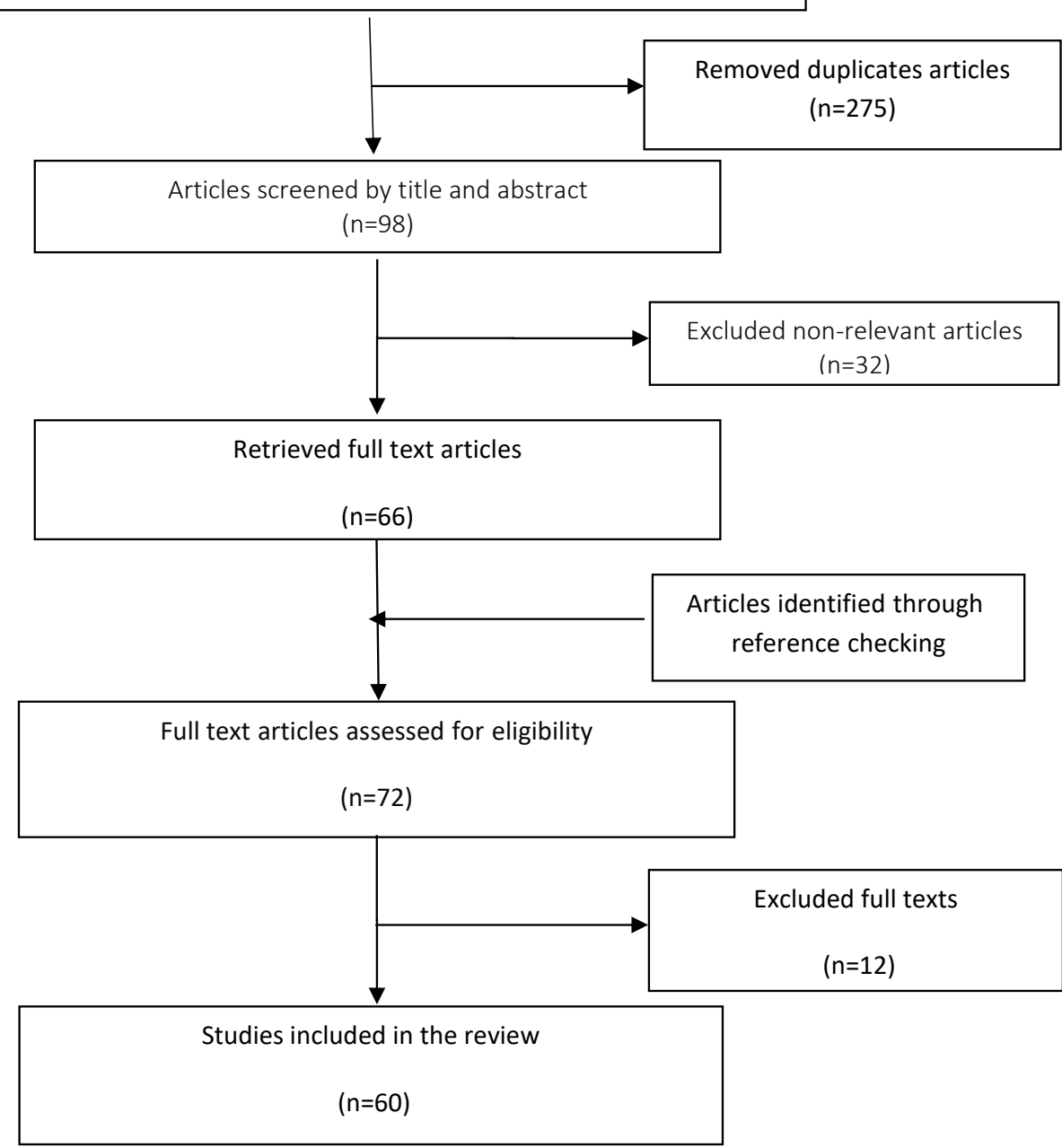

Figure 1. Flowchart of study selection

Journal of Pediatrics Review

\section{Results}

Most of the studies have cross-sectional, case-control, and prospective design. Most of them evaluated the possible risk factors for primary $\mathrm{CH}$. Reported risk factors for transient $\mathrm{CH}$ were as follows: iodine deficiency or excess, prematurity, old maternal age, male gender, retinopathy of prematurity, twin pregnancy, maternal autoimmune thyroid disease, intrauterine growth retardation, and cesarean delivery $(19,24,26,33,51,56,63)$. Reported risk factors for permanent $\mathrm{CH}$ with dysgenesis of the thyroid gland were as follows: female gender, familial history of $\mathrm{CH}$, birth in geographical areas with a high rate of the disease, old maternal age, ethnicity (Caucasians) but not seasonality $(5,15,42,50,56,61)$.
Reported risk factors for permanent $\mathrm{CH}$ with dyshormonogenesis were the familial history of $\mathrm{CH}$ and origin of both parents from the high-risk geographical region (15). In five papers, the effects of risk factors on the TSH level during screening were evaluated $(14,25,28,6,33)$.

\section{Discussion}

In this review study, we studied all reported studies in the field of $\mathrm{CH}$ risk factors. Most of the reviewed studies were cross-sectional and evaluated the risk factors of primary $\mathrm{CH}$. Few studies were investigating the risk factors of permanent vs. transient or different etiologies of $\mathrm{CH}$. Though some of the reported risk factors for permanent and temporary $\mathrm{CH}$ and various etiologies of permanent $\mathrm{CH}$ were similar, some of them were specific for the mentioned groups. By considering the reported group differences, we could design more studies for better understand- 
Table 1. Details of the papers

\begin{tabular}{|c|c|c|c|c|c|}
\hline No. & $\begin{array}{c}\text { Name of First Author, } \\
\text { Year, Place }\end{array}$ & Sample Size & Type of Study & Type of $\mathrm{CH}$ & Reported Risk Factors \\
\hline 1 & $\begin{array}{l}\text { Thalhammer et al. } \\
\text { Austria (13) }\end{array}$ & $\begin{array}{c}\text { Results of CH screening since } \\
1976\end{array}$ & Cross-sectional & Permanent $\mathrm{CH}$ & Seasonality \\
\hline 2 & $\begin{array}{l}\text { Meberg A et al. } \\
\text { Norway (14) }\end{array}$ & $\begin{array}{c}46 \text { smoker and } 49 \text { nonsmoker } \\
\text { mothers }\end{array}$ & Case-control & Serum TSH & Smoking mother \\
\hline 3 & $\begin{array}{l}\text { Rosenthal et al. } \\
\text { England (69) }\end{array}$ & $\begin{array}{l}289697 \text { screened neonates } \\
\text { (from November } 1981 \text { to } \\
\text { February 1987) }\end{array}$ & Cross-sectional & Primary $\mathrm{CH}$ & Parental consanguinity \\
\hline 3 & $\begin{array}{l}\text { Virtanen et al. } \\
\text { Finland (15) }\end{array}$ & 307000 screened neonates & Cross-sectional & Permanent $\mathrm{CH}$ & $\begin{array}{l}\text { Female gender, } \mathrm{CH} \text { in the family, } \\
\text { high risk geographic region, sea- } \\
\text { sonality for dysgenesis } \\
\text { History of } \mathrm{CH} \text { in the family and } \\
\text { high risk geographic region for } \\
\text { dyshormonogenesis }\end{array}$ \\
\hline 4 & $\begin{array}{l}\text { Kaiserman et al. } \\
\text { Israel (16) }\end{array}$ & 303 primary $\mathrm{CH}$ patients & Cross-sectional & Primary $\mathrm{CH}$ & - \\
\hline 5 & $\begin{array}{l}\text { Lorev et al. } \\
\text { USA, California (17) }\end{array}$ & Over 5 million infants & Prospective & Primary $\mathrm{CH}$ & $\begin{array}{l}\text { Female gender for all ethnic } \\
\text { groups except blacks }\end{array}$ \\
\hline 6 & $\begin{array}{l}\text { Sorcini et al. } \\
\text { Italy (18) }\end{array}$ & 239 cases of $\mathrm{CH}$ & Prospective & Primary $\mathrm{CH}$ & lodine deficiency \\
\hline 7 & $\begin{array}{l}\text { Dussault et al. } \\
\text { Quebec, Canada (19) }\end{array}$ & 259 mothers of $\mathrm{CH}$ newborns & Cross-sectional & Transient $\mathrm{CH}$ & $\begin{array}{l}\text { Maternal autoimmune thyroid dis- } \\
\text { ease ( antimicrosomal antibodies) }\end{array}$ \\
\hline 8 & $\begin{array}{l}\text { Hall et al., } \\
\text { England (20) }\end{array}$ & $\begin{array}{c}1128632 \text { neonates screened } \\
\text { over } 16 \text { years }\end{array}$ & Cross-sectional & Primary $\mathrm{CH}$ & Season, Parental consanguinity \\
\hline 9 & $\begin{array}{c}\text { Waller et al. } \\
\text { California,USA (12) }\end{array}$ & 1806 cases of $\mathrm{CH}$ & Cross-sectional & Primary $\mathrm{CH}$ & $\begin{array}{l}\text { Low-birth weight, macrosomia, } \\
\text { ethnicity, gender }\end{array}$ \\
\hline 10 & $\begin{array}{l}\text { Rocchi et al. } \\
\text { Italy (21) }\end{array}$ & $92 \mathrm{CH}$ patients & Retrospective & Primary $\mathrm{CH}$ & - \\
\hline 11 & $\begin{array}{c}\text { Henry et al. } \\
\text { Saudi Arabia (22) }\end{array}$ & $\begin{array}{l}44 \mathrm{CH} \text { patients from } 121404 \\
\text { screened neonates }\end{array}$ & Cross-sectional & Primary $\mathrm{CH}$ & - \\
\hline 12 & $\begin{array}{l}\text { Ordookhani et al. } \\
\text { Tehran, Iran (23) }\end{array}$ & $\begin{array}{l}22 \mathrm{CH} \text { patients from } 20107 \\
\text { screened neonates }\end{array}$ & Cross-sectional & Primary $\mathrm{CH}$ & Parental consanguinity \\
\hline 13 & $\begin{array}{l}\text { Buyukgebiz A, } \\
\text { Turkey (24) }\end{array}$ & - & Review paper & Transient $\mathrm{CH}$ & Prematurity \\
\hline 14 & $\begin{array}{l}\text { Ouhoummane et al. } \\
\text { Canada (25) }\end{array}$ & $\begin{array}{l}32978 \text { screened newborns } \\
\text { (1993-1999) }\end{array}$ & Retrospective & Primary $\mathrm{CH}$ & $\begin{array}{l}\text { Chlorine dioxide }(\mathrm{ClO} 2) \text { in disin- } \\
\text { fected water for } \\
\text { Low-birth-weight infants }\end{array}$ \\
\hline 15 & $\begin{array}{l}\text { Ordookhani et al. } \\
\text { Tehran, Iran (26) }\end{array}$ & $\begin{array}{l}41 \mathrm{CH} \text { patients }(6 \text { of them } \\
\text { transient } \mathrm{CH})\end{array}$ & Cross-sectional & Transient $\mathrm{CH}$ & $\begin{array}{l}\text { Exposure with iodinated disinfec- } \\
\text { tants during the perinatal period }\end{array}$ \\
\hline 16 & $\begin{array}{l}\text { Lian et al. } \\
\text { China (27) }\end{array}$ & $35 \mathrm{CH}$ patients & Retrospective & Primary $\mathrm{CH}$ & $\begin{array}{l}\text { Prematurity, modest or massive } \\
\text { hypertension during pregnancy, } \\
\text { high serum anti-thyroid peroxidase } \\
\text { antibodies levels }\end{array}$ \\
\hline 17 & $\begin{array}{l}\text { McElduff et al. } \\
\text { Australia (28) }\end{array}$ & 2031screened neonates & Cohort study & Primary $\mathrm{CH}$ & Cesarean delivery \\
\hline 18 & $\begin{array}{l}\text { Medda et al. } \\
\text { Italy (6) }\end{array}$ & $\begin{array}{l}173 \text { cases and } 690 \text { controls } \\
\text { were enrolled in } 4 \text { years }\end{array}$ & Case-control & $\begin{array}{c}\text { Permanent } \\
\text { and transient } \\
\mathrm{CH}\end{array}$ & $\begin{array}{l}\text { Twin birth, birth defects, female } \\
\text { gender and gestational age }>40 \\
\text { weeks, family history of thyroid } \\
\text { diseases among parents, maternal } \\
\text { diabetes for permanent } \mathrm{CH} \\
\text { intrauterine growth retardation, } \\
\text { prematurity for transient } \mathrm{CH}\end{array}$ \\
\hline 19 & $\begin{array}{l}\text { Deladoëy et al. } \\
\text { Québec, Canada (5) }\end{array}$ & $424 \mathrm{CH}$ patients & Cross-sectional & $\begin{array}{l}\text { Permanent } \mathrm{CH} \\
\text { with dysgen- } \\
\text { esis }\end{array}$ & - \\
\hline
\end{tabular}




\begin{tabular}{|c|c|c|c|c|c|}
\hline No. & $\begin{array}{l}\text { Name of First Author, } \\
\text { Year, Place }\end{array}$ & Sample Size & Type of Study & Type of CH & Reported Risk Factors \\
\hline 20 & $\begin{array}{l}\text { Ordookhani et al. } \\
\text { Tehran, Iran (29) }\end{array}$ & 48106 screened neonates & Cross-sectional & Serum TSH & Cesarean delivery \\
\hline 21 & $\begin{array}{l}\text { Hashemipour et al. } \\
\text { Isfahan, Iran (30) }\end{array}$ & $\begin{array}{l}358 \mathrm{CH} \text { patients from } 113282 \\
\text { screened neonates }\end{array}$ & Cross-sectional & Primary $\mathrm{CH}$ & $\begin{array}{l}\text { The month of birth, suspected } \\
\text { environmental factors }\end{array}$ \\
\hline 22 & $\begin{array}{l}\text { Gu et al. } \\
\text { Japan (31) }\end{array}$ & $1586 \mathrm{CH}$ patients & Cross-sectional & Primary $\mathrm{CH}$ & Gender, season \\
\hline 23 & $\begin{array}{l}\text { Hashemipour et al. } \\
\text { Isfahan, Iran (32) }\end{array}$ & $274 \mathrm{CH}$ patients & Retrospective & Primary $\mathrm{CH}$ & First cousin parental consanguinity \\
\hline 24 & $\begin{array}{l}\text { Olivieri et al. } \\
\text { Italy (33) }\end{array}$ & $3600 \mathrm{CH}$ patients & Retrospective & $\begin{array}{l}\text { Both transient } \\
\text { and perma- } \\
\text { nent } \mathrm{CH}\end{array}$ & Twin birth, environmental factors \\
\hline 25 & $\begin{array}{l}\text { Mao et al. } \\
\text { China (9) }\end{array}$ & $289 \mathrm{CH}$ patients & Cross-sectional & Primary $\mathrm{CH}$ & $\begin{array}{l}\text { Post-term birth, low-birth-weight } \\
\text { infants, macrosomia }\end{array}$ \\
\hline 26 & $\begin{array}{l}\text { Rowland et al. } \\
\text { USA (34) }\end{array}$ & - & Clinical inquiries & Primary $\mathrm{CH}$ & $\begin{array}{l}\text { Prematurity, infants wellbeing (ce- } \\
\text { rebral pathology, low Apgar scores, } \\
\text { respiratory distress syndrome, } \\
\text { persistent ductus arteriosus requir- } \\
\text { ing treatment, necrotizing entero- } \\
\text { colitis), maternal thyroid disease, } \\
\text { iodine deficiency/excess }\end{array}$ \\
\hline 27 & $\begin{array}{l}\text { Eftekhari et al. } \\
\text { Kerman, Iran (35) }\end{array}$ & $\begin{array}{l}23 \mathrm{CH} \text { patients from } 3000 \\
\text { screened neonates }\end{array}$ & Cross-sectional & Primary $\mathrm{CH}$ & $\begin{array}{l}\text { Gender, maternal age, families } \\
\text { socioeconomic condition, parents } \\
\text { education, mothers iodinated salt } \\
\text { consumption, parents' occupation, } \\
\text { thyroid hormone used by mothers }\end{array}$ \\
\hline 28 & $\begin{array}{l}\text { Sepandi et al. } \\
\text { Shiraz, Iran (36) }\end{array}$ & $\begin{array}{l}126 \mathrm{CH} \text { patients and } 401 \\
\text { controls }\end{array}$ & Case-control & Primary $\mathrm{CH}$ & $\begin{array}{l}\text { Parental consanguinity, birth } \\
\text { defects, birth defects in the first- } \\
\text { degree relatives, female gender, } \\
\text { twin births, prematurity }\end{array}$ \\
\hline 29 & $\begin{array}{l}\text { Cranston et al. } \\
\text { California, USA (37) }\end{array}$ & $698 \mathrm{CH}$ patients & Cross-sectional & Primary $\mathrm{CH}$ & $\begin{array}{l}\text { Prematurity, maternal age, civilian } \\
\text { maternal status }\end{array}$ \\
\hline 30 & $\begin{array}{l}\text { Hashemipour et al. } \\
\text { Isfahan, Iran (38) }\end{array}$ & $\begin{array}{l}68 \mathrm{CH} \text { and } 178 \text { healthy chil- } \\
\text { dren and their mothers }\end{array}$ & Cross-sectional & Primary $\mathrm{CH}$ & $\begin{array}{l}\text { Milk iodine concentration and } \\
\text { iodine excess }\end{array}$ \\
\hline 31 & $\begin{array}{l}\text { Hinton et al. } \\
\text { The USA ( } 8 \text { ) }\end{array}$ & $\begin{array}{l}142 \mathrm{CH} \text { patients from } 47075 \\
\text { screened neonates }\end{array}$ & Cross-sectional & Primary $\mathrm{CH}$ & $\begin{array}{c}\text { Race, ethnicity, sex, and pregnancy } \\
\text { outcomes }\end{array}$ \\
\hline 32 & $\begin{array}{l}\text { Aminzadeh et al. } \\
\text { Ahvaz, Iran (39) }\end{array}$ & $\begin{array}{l}142 \mathrm{CH} \text { patients from } 47075 \\
\text { screened neonates }\end{array}$ & $\begin{array}{l}\text { A prospective } \\
\text { two-year study }\end{array}$ & Permanent $\mathrm{CH}$ & Season \\
\hline 33 & $\begin{array}{l}\text { Hashemipour et al. } \\
\text { Isfahan, Iran (40) }\end{array}$ & $\begin{array}{l}194 \mathrm{CH} \text { and } 350 \text { normal and } \\
\text { their first-degree relatives }\end{array}$ & Case-control & Primary $\mathrm{CH}$ & Hypothyroidism \\
\hline 34 & $\begin{array}{l}\text { Safar Alizade et al. } \\
\text { Khoy, Iran (41) }\end{array}$ & $16 \mathrm{CH}$ patients & Prospective & Primary $\mathrm{CH}$ & $\begin{array}{l}\text { Parental consanguinity, maternal } \\
\text { diet during pregnancy (chicken) }\end{array}$ \\
\hline 35 & $\begin{array}{l}\text { Stoppa-Vaucher et al. } \\
\text { Montréal, Canada (42) }\end{array}$ & $\begin{array}{c}190 \text { patients with TD ( } 147 \\
\text { ectopies, } 40 \text { athyreosis, and } \\
3 \text { hypoplasias) and the } 44 \\
\text { patients with DH }\end{array}$ & Case-control & $\begin{array}{l}\text { Permanent } \mathrm{CH} \\
\text { with Thyroid } \\
\text { Dysgenesis } \\
\text { (TD) }\end{array}$ & Ethnicity \\
\hline 36 & $\begin{array}{l}\text { Hashemipour et al. } \\
\text { Isfahan, Iran (45) }\end{array}$ & $\begin{array}{l}65 \text { patients with } \mathrm{CH} \text { and their } \\
\text { mothers as the case group } \\
\text { and } 148 \text { healthy neonates and } \\
\text { their mothers as the control } \\
\text { group }\end{array}$ & Case-control & Primary $\mathrm{CH}$ & $\begin{array}{c}\text { Maternal thyroid autoimmunity } \\
\text { (Thyrotropin Receptor Antibodies } \\
{[\text { TRAb]) }}\end{array}$ \\
\hline 37 & $\begin{array}{l}\text { Zeinalzadeh et al. } \\
\text { East Azerbaijan, Iran (44) }\end{array}$ & $\begin{array}{l}94 \mathrm{CH} \text { patients from } 62,459 \\
\text { screened neonates }\end{array}$ & Cross-sectional & Primary $\mathrm{CH}$ & Maternal age \\
\hline 38 & Ooki S.Japan (45) & $18 \mathrm{CH}$ patients & Retrospective & Primary $\mathrm{CH}$ & Multiple births \\
\hline 39 & $\begin{array}{l}\text { Abdelmoktader et al. } \\
\text { Egypt (46) }\end{array}$ & $\begin{array}{l}320 \text { cases and } 320 \text { controls } \\
\text { enrolled in } 8 \text { years }\end{array}$ & $\begin{array}{l}\text { A population- } \\
\text { based case-con- } \\
\text { trol study }\end{array}$ & Primary $\mathrm{CH}$ & $\begin{array}{l}\text { Twin birth, birth defects, female } \\
\text { gender, gestational age }>40 \text { weeks, } \\
\text { and gestational diabetes }\end{array}$ \\
\hline
\end{tabular}




\begin{tabular}{|c|c|c|c|c|c|}
\hline No. & $\begin{array}{l}\text { Name of First Author, } \\
\text { Year, Place }\end{array}$ & Sample Size & Type of Study & Type of CH & Reported Risk Factors \\
\hline 40 & $\begin{array}{l}\text { Rezaeian et al. } \\
\text { Hamadan, Iran (47) }\end{array}$ & $\begin{array}{l}1313 \text { enrolled neonates, } 277 \\
\text { (159 girls) were cases, and } \\
1036 \text { (531 girls) were controls }\end{array}$ & Case-control & Primary $\mathrm{CH}$ & $\begin{array}{l}\text { Twin birth, birth season, maturity, } \\
\text { jaundice at birth, birth weight, age } \\
\text { at pregnancy, maternal anemia } \\
\text { and goiter, gestational age, deliv- } \\
\text { ery type, father's education and } \\
\text { smoking status, and consanguinity }\end{array}$ \\
\hline 41 & $\begin{array}{l}\text { Ng et al. } \\
\text { Liverpool, UK (48) }\end{array}$ & $\begin{array}{l}6498 \text { neonates during } \mathrm{CH} \\
\text { screening }\end{array}$ & Retrospective & Primary $\mathrm{CH}$ & Low-birth weight \\
\hline 42 & $\begin{array}{l}\text { Dalili et al. } \\
\text { Guilan, Iran (49) }\end{array}$ & $\begin{array}{l}221 \mathrm{CH} \text { patients from } \\
119701 \text { screened neonates }\end{array}$ & Retrospective & Primary $\mathrm{CH}$ & $\begin{array}{l}\text { Low-birth weight, postdate deliv- } \\
\text { ery, macrosomia, vaginal delivery }\end{array}$ \\
\hline 43 & $\begin{array}{l}\text { Kirmızibekmez et al. } \\
\text { Turkey (50) }\end{array}$ & $234 \mathrm{CH}$ patients & Retrospective & $\begin{array}{l}\text { Permanent } \mathrm{CH} \\
\text { with dysgen- } \\
\text { esis }\end{array}$ & Maternal age \\
\hline 44 & $\begin{array}{l}\text { Rabbiosi et al. } \\
\text { Italy (51) }\end{array}$ & $\begin{array}{l}84 \mathrm{CH} \text { patients and ectopic } \\
\text { thyroid gland }\end{array}$ & Prospective & $\begin{array}{c}\text { Permanent } \\
\text { and transient } \\
\mathrm{CH}\end{array}$ & $\begin{array}{c}\text { Prematurity, first-degree familial } \\
\text { history of goiter/nodules for per- } \\
\text { manent } \mathrm{CH} \text {, mild iodine deficiency } \\
\text { for transient } \mathrm{CH}\end{array}$ \\
\hline 45 & $\begin{array}{l}\text { Esmailnasab et al. } \\
\text { Kordestan, Iran (52) }\end{array}$ & $\begin{array}{l}105 \mathrm{CH} \text { patients and } 105 \\
\text { controls }\end{array}$ & $\begin{array}{l}\text { Case-control } \\
\text { study }\end{array}$ & Primary $\mathrm{CH}$ & Familial thyroid disease \\
\hline 46 & $\begin{array}{c}\text { Rezaeian et al. } \\
\text { Hamadan, Iran (53) }\end{array}$ & $\begin{array}{l}277 \text { cases ( } \mathrm{CH} \text { patients) and } \\
1036 \text { controls }\end{array}$ & Case-control & Primary $\mathrm{CH}$ & $\begin{array}{l}\text { Interaction of gender (girl) and } \\
\text { birth season (summer) }\end{array}$ \\
\hline 47 & $\begin{array}{l}\text { Dorreh et al. } \\
\text { Arak, Iran (54) }\end{array}$ & $\begin{array}{l}414 \mathrm{CH} \text { patients from } 127112 \\
\text { screened neonates }\end{array}$ & Cross-sectional & Primary $\mathrm{CH}$ & Family history of thyroid diseases \\
\hline 48 & $\begin{array}{l}\text { Uenaka et al. } \\
\text { Japan (55) }\end{array}$ & $\begin{array}{l}35 \text { pregnancies complicated by } \\
\text { Graves' disease, } \\
9 \text { cases with neonatal thyroid } \\
\text { dysfunction and } 22 \text { with nor- } \\
\text { mal thyroid function }\end{array}$ & Prospective & Primary $\mathrm{CH}$ & Maternal FT4 level \\
\hline 49 & $\begin{array}{l}\text { Fan et al. } \\
\text { China (56) }\end{array}$ & $1210 \mathrm{CH}$ patients & Prospective & Transient $\mathrm{CH}$ & lodine deficiency \\
\hline 50 & $\begin{array}{l}\text { Satoh et al. } \\
\text { Japan (57) }\end{array}$ & $\begin{array}{l}212 \text { infants born to mothers } \\
\text { who become pregnant after } \\
\text { undergoing hysterosalpin- } \\
\text { gography involving the use of } \\
\text { ethiodized oil }\end{array}$ & Prospective & Primary $\mathrm{CH}$ & $\begin{array}{l}\text { Using ethiodized oil contrast } \\
\text { medium during hysterosalpingog- } \\
\text { raphy }\end{array}$ \\
\hline 51 & $\begin{array}{l}\text { Mehrnejat et al. } \\
\text { Isfahan, Iran (58) }\end{array}$ & $\begin{array}{l}667 \mathrm{CH} \text { patients from } 275485 \\
\text { screened neonates }\end{array}$ & $\begin{array}{l}\text { Descriptive- } \\
\text { analytic }\end{array}$ & Primary $\mathrm{CH}$ & $\begin{array}{l}\text { Nitrate concentration in drinking- } \\
\text { water }\end{array}$ \\
\hline 52 & $\begin{array}{l}\text { Zhou et al. } \\
\text { China (59) }\end{array}$ & $\begin{array}{l}125 \text { neonates with } \mathrm{CH} \text { (case } \\
\text { group) and } 375 \text { neonates } \\
\text { without } \mathrm{CH} \text { (control group) }\end{array}$ & Case-control & Primary $\mathrm{CH}$ & $\begin{array}{l}\text { Mother's age, gestational diabetes, } \\
\text { gestational thyroid disease, birth } \\
\text { weight, gestational age, fetus num- } \\
\text { ber, fetal distress, birth defects }\end{array}$ \\
\hline 53 & $\begin{array}{l}\text { Trumpff et al. } \\
\text { Brussels, Belgium (60) }\end{array}$ & $\begin{array}{l}313 \text { Belgian mothers and their } \\
\text { 4- to 5-year-old children }\end{array}$ & $\begin{array}{l}\text { Retrospective } \\
\text { cohort study }\end{array}$ & Primary $\mathrm{CH}$ & $\begin{array}{l}\text { Season, maternal smoking, lower } \\
\text { weight gain during pregnancy, } \\
\text { gestational age }\end{array}$ \\
\hline 54 & $\begin{array}{l}\text { Dayal et al. } \\
\text { India (61) }\end{array}$ & $80 \mathrm{CH}$ patients & Retrospective & $\begin{array}{l}\text { Permanent } \mathrm{CH} \\
\text { (dysgenesis) }\end{array}$ & Maternal age \\
\hline 55 & $\begin{array}{l}\text { Keshavarzian et al. } \\
\text { Shadegan, Iran (62) }\end{array}$ & $\begin{array}{c}203 \mathrm{CH} \text { patients and } 657 \\
\text { controls }\end{array}$ & Case-control & Primary $\mathrm{CH}$ & $\begin{array}{c}\text { Parental consanguinity, urbaniza- } \\
\text { tion }\end{array}$ \\
\hline 56 & $\begin{array}{l}\text { Aguiar et al. } \\
\text { Massachusetts, } \\
\text { USA (63) }\end{array}$ & 76 & Retrospective & $\begin{array}{l}\text { Transient vs. } \\
\text { permanent } \mathrm{CH}\end{array}$ & $\begin{array}{c}\text { Maternal age, cesarean delivery, } \\
\text { retinopathy of prematurity for } \\
\text { transient } \mathrm{CH}\end{array}$ \\
\hline 57 & $\begin{array}{c}\text { Blasig et al. } \\
\text { Berlin, Germany (66) }\end{array}$ & $84 \mathrm{CH}$ patients & Cross-sectional & Primary $\mathrm{CH}$ & Serum Cu \\
\hline 58 & $\begin{array}{l}\text { Yang et al. } \\
\text { China (67) }\end{array}$ & $\begin{array}{l}\mathrm{CH} \text { patients diagnosed during } \\
25 \text { years of } \mathrm{CH} \text { screening }\end{array}$ & Cross-sectional & Primary $\mathrm{CH}$ & $\begin{array}{l}\text { Female sex, preterm birth, older } \\
\text { gestational age, low-birth weight, } \\
\text { and preterm birth }\end{array}$ \\
\hline 59 & $\begin{array}{l}\text { Anastasovska et al. } \\
\text { Macedonia (68) }\end{array}$ & $46 \mathrm{CH}$ patients & $\begin{array}{l}\text { A 14-year retro- } \\
\text { spective cohort } \\
\text { analysis }\end{array}$ & Primary $\mathrm{CH}$ & Ethnicity \\
\hline
\end{tabular}




\begin{tabular}{cccccc}
\hline No. & $\begin{array}{c}\text { Name of First Author, } \\
\text { Year, Place }\end{array}$ & Sample Size & Type of Study & Type of CH & Reported Risk Factors \\
\hline 60 & $\begin{array}{c}\text { Khanjani et al. } \\
\text { Kerman, Iran (69) }\end{array}$ & $\begin{array}{c}773 \mathrm{CH} \text { patients from 288437 } \\
\text { screened neonates }\end{array}$ & Cross-sectional & Primary CH & Season \\
\hline
\end{tabular}

ing of different subgroups of $\mathrm{CH}$. As mentioned previously, though there were studies regarding $\mathrm{CH}$-related risk factors $(6,34,36,44,47,49,56,59,63)$ there was no comprehensive review in this field. Moreover, for some important risk factors such as seasonality or gender differences, the results of studies were not in agreement. We classified the risk factors in the following categories; known risk factors with enough and appropriate evidence, known risk factors with controversial results, and risk factors with limited evidence which need more evaluations.

The role of some risk factors such as ethnicity, thyroid disorders in families, other birth defects, pre-term and post-term delivery, low- and high-birth weight, parental consanguinity and twin or multiple pregnancies for $\mathrm{CH}$ have been clearly determined in many studies $(12,13$, $20,23-25,32,33,45,48,52,54,55,62,65,66)$. Though there were also few studies which did not report such an association, almost all of them support the role of above-mentioned risk factors for $\mathrm{CH}$. However, the additive effect of the risk factors for the occurrence of $\mathrm{CH}$ should be investigated in future research.

lodine deficiency or excess $(18,26,34)$, gender (31, $17)$, seasonality $(5,13,20-22,30,67)$ maternal age $(12$, $37,44,61,63)$, type of delivery $(28,62)$ and maternal anti-thyroid drug use $(6,27)$ were the risk factors with controversial reports. Though their role as $\mathrm{CH}$-related risk factors has been demonstrated in previous studies, the findings are not conclusive.

lodine deficiency is one of the most critical risk factors for $\mathrm{CH}$, but by the elimination of iodine deficiency in different countries, it seems that iodine excess is considered as a risk factor for $\mathrm{CH}(18,26,34)$. lodine excess could be a result of different factors such as using iodinated salt and different pharmacological agents using for therapeutic or diagnostic procedures in specific disorders.

Satoh et al. in Japan evaluated the rate of thyroid dysfunction in neonates born to mothers who have undergone Hysterosalpingography (HSG) involving an oilsoluble iodinated contrast medium. According to their findings in the thyroid dysfunction group, the median dosage of ethiodized oil was significantly higher than in the normal thyroid function group. They recommended that when infertile women undergo HSG, the administrated dosage of oil-soluble iodinated contrast medium should be reduced to minimize the risk of thyroid dysfunction in fetus or neonates (57).

Previous studies showed an association between gender and $\mathrm{CH}$. Many reports have indicated that $\mathrm{CH}$ is frequently found in girls $(12,15,17,6,31,36,44,46,53,56)$. According to previous studies, the female to male ratio was approximately 1.0 among hereditary cases of $\mathrm{CH}$ (31). Moreover, this ratio was about 2.0 for the $\mathrm{CH}$ cases with both athyreosis and ectopic groups (17). Castanet et al. reported that the female preponderance over males for isolated $\mathrm{CH}$ was similar to those with the ectopic thyroid gland or athyreosis (68). Accordingly, the preponderance of female gender for $\mathrm{CH}$ is mainly related to thyroid dysgenesis. These results were also reported in another study (15). According to our findings, girls were at higher risk of $\mathrm{CH}$ than boys. But there are also studies which did not show such an association (49).

Recently, Rezaeian et al. in Hamedan, Iran studied the potential interactions that could change the effect of gender on congenital hypothyroidism (53). They indicated that odds ratio estimates of $\mathrm{CH}$ for investigated factors (except for birth season) did not differ substantially between girls and boys. Similarly, Ng et al. found no significant difference between girls and boys regarding gestation and birth weight in all etiological subgroups such as athyreosis and ectopic groups (48). Rezaeian and colleagues have finally indicated that birth season might act as an interaction to increase the risk of $\mathrm{CH}$ in girls (53).

However, it is unclear why girls have a higher incidence rate of $\mathrm{CH}$ than boys, while there is no difference in the proportion of other risk factors between them. So, the reasons for gender differences deserve further investigations. The results of the reviewed literature regarding the seasonal relationship were inconsistent, too. Gu et al. in Japan reported that temperature and season had a significant effect on $\mathrm{CH}$. According to them, from January to December, males and females had one and two peaks, respec- 
tively (30). In the British Midland, higher incidence of $\mathrm{CH}$ was reported in fall between October and December (20).

Some studies did not report any seasonal pattern for $\mathrm{CH}$. Rosenthal et al. observed no seasonal difference in the incidence of $\mathrm{CH}$ in the Northwest of England, in Asian families compared with non-Asians (69). No evidence of seasonal variations was reported during the $\mathrm{CH}$ screening program in Saudi Arabia and Italy $(21,22)$. Kaiserman et al. in Israel conducted a 10-year temporal analysis of primary $\mathrm{CH}$; the average monthly incidence showed a small peak in August, but, monthly incidence of $\mathrm{CH}$ had no significant periodicity (16).

There were different studies from Iran on this topic, too. Ordookhani et al. reported a significant correlation between winter and $\mathrm{CH}$. Hashemipour et al. reported higher and lower incidence rates of $\mathrm{CH}$ in summer and the last month of autumn, respectively (16). Their findings were not similar to others. They suggested that other factors such as exposure to different chemical compounds, seasonal environmental factors, and differences in climate might play a role in the etiology of $\mathrm{CH}$.

In previous studies in Iran, Aminzadeh et al. investigated the association between seasonal changes in temperature and the prevalence of congenital Hypothyroidism $(\mathrm{CH})$ in Southwest Iran and reported that the prevalence of $\mathrm{CH}$ had a significant negative correlation with temperature. The odds of being affected increased by $4 \%$ for each $1^{\circ} \mathrm{C}$ drop in temperature (39). Findings of other studies from Iran showed a higher incidence of $\mathrm{CH}$ in autumn and winter.

The impact of environmental factors such as climatic conditions and seasonal changes in the incidence of $\mathrm{CH}$ is still unclear. In a recent study in Iran, Khanjani et al. for the first time evaluated the effects of several climatic factors such as temperature, humidity, and rainfall on the incidence of $\mathrm{CH}$. They did not find any significant association between $\mathrm{CH}$ and climate factors, in Kerman Province, whereas they reported the highest rate of $\mathrm{CH}$ in October (autumn) and lowest in June (summer) (67).

It seems that the reported discrepancy may be due to differences in climate, living conditions, and various levels of iodine in different geographical areas. It is also suggested that different environmental and genetic factors could interact with seasonality and consequently could affect the incidence of $\mathrm{CH}$ in each region.

Some studies reported advanced maternal age as a risk factor for $\mathrm{CH}(12,37,44)$. But some of them have reported such an association only for thyroid dysgenesis (64). According to the documents, the maternal age of more than 35 years could be a risk factor for $\mathrm{CH}$ (47).

Type of delivery was another conflicting risk factor. McElduff et al. in their investigation among 2031 infants have indicated that TSH levels were greater among babies delivered by cesarean section (28). Rezaeian and colleagues have also reported a higher incidence rate of $\mathrm{CH}$ in both emergency and elective cesarean sections (47). Whereas Ordookhani et al. reported that umbilical cord blood TSH and rates of hyperthyrotropinemia are lower in cesarean section than in vaginal deliveries. They showed that povidoneiodine disinfection at delivery has an effect neither on TSH concentrations nor on the rate of hyperthyrotropinemia in the iodine-replete area of Iran (6).

Similarly, Dalili et al. have reported that the frequency of Normal Vaginal Delivery (NVD) was significantly higher in neonates with $\mathrm{CH}$ compared to the normal population (49). It seems that different conditions related to the type of delivery, including the iodine condition of the population, method of delivery and using different disinfectant have an impact on the association of type of delivery and $\mathrm{CH}$ occurrence.

Some studies reported that maternal anti-thyroid drug use and its pattern could affect thyroid function of neonates $(27,55)$. Lian et al. in China reported that the risk of abnormal thyroid function of infants whose hyperthyroid mothers did not take anti-thyroid drugs until the third trimester of pregnancy might be increased (26). In one study, using thyroid hormones by mother was not considered as a risk factor for $\mathrm{CH}$.

Some of the reported risk factors, including environmental pollutants $(25,58)$, dietary component of mothers during prenatal period $(41,64)$, neonatal jaundice (47), maternal anemia (48), intrauterine growth retardation (6), lower weight gain during pregnancy (60), urbanization (62), parental occupation and education (35, $47)$, gestational diabetes $(6,46,59)$, and smoking (14, $47,60)$ have limited evidence. It seems that more studies for investigating the association of the mentioned risk factors with $\mathrm{CH}$ are necessary. Of the above-mentioned risk factors, some have high priority, including environmental pollutants, smoking, gestational diabetes, and maternal anemia due to their effectiveness in preventative medicine.

So far, few studies have investigated the effect of environmental factors on $\mathrm{CH}$ incidence. Ouhoummane et al. in Cana- 
da compared the thyroid function of newborns from $11 \mathrm{mu}-$ nicipalities where drinking water was disinfected by Chlorine Dioxide $\left(\mathrm{ClO}_{2}\right)$ with that of newborns from 15 municipalities using chlorine disinfection. There was no significant increase in the TSH level and rate of $\mathrm{CH}$ when all newborns exposed to $\mathrm{ClO}_{2}$ were considered. However, for newborns with lowbirth weight, mean TSH level was significantly higher among those exposed to $\mathrm{ClO}_{2}$ than for those in the reference group. They concluded that $\mathrm{ClO}_{2}$ was a risk factor for $\mathrm{CH}$ in preterm and low-birth-weight neonates (26). In another study in Iran, Mehrnejat et al. found no significant relationship between nitrate concentration in drinking water and the incidence of $\mathrm{CH}$ through linear regression analysis (58).

In two studies, the dietary component of mothers has been reported as risk factors for $\mathrm{CH}$, including $\mathrm{Cu}$ deficiency and some other nutritional components (41, 64). It seems that evaluating the association of prenatal dietary components is helpful for identification of $\mathrm{CH}$ related modifiable risk factors. The limitation of the current review was the heterogeneity of papers so that we could not do meta-analysis in this field. The strength of this review was its novelty. There was not any systematic review regarding the risk factors of $\mathrm{CH}$.

\section{Conclusions}

The findings of the current review provide us basic information about reported $\mathrm{CH}$-related risk factors from different countries. Using this information, we could plan more etiologic studies to investigate the pathogenesis of the disorder, design interventional studies for the known modifiable risk factors, and reduce the rate of $\mathrm{CH}$ in our region. Besides, for risk factors with limited evidence, more studies should be performed.

Moreover, the discrepancies between different studies regarding $\mathrm{CH}$-related risk factors may also be due to the interaction of different risk factors in different populations with different genetic background and different environmental factors. Also, neonatal, maternal, and pregnancy-related determinants are responsible for the occurrence of $\mathrm{CH}$, which should be investigated through more complex statistical analysis.

\section{Ethical Considerations}

\section{Compliance with ethical guidelines}

There are no ethical considerations to be noted in this article.
Funding

This research did not receive any specific grant from funding agencies in the public, commercial, or not-forprofit sectors.

\section{Authors' contributions}

All authors contributed in designing, running, and writing all parts of the research.

\section{Conflicts of interest}

The authors declare no conflict of interest.

\section{References}

1. Rastogi MV, LaFranchi SH. Congenital hypothyroidism. Orphanet Journal of Rare Diseases. 2010; 5:17. [DOI:10.1186/1750-1172-5-17] [PMID] [PMCID]

2. Wassner AJ, Brown RS. Congenital hypothyroidism: Recent advances. Current Opinion in Endocrinology, Diabetes and Obesity. 2015; 22(5):407-12. [DOI:10.1097/ MED.0000000000000181] [PMID]

3. Heindel JJ, Zoeller RT. Thyroid hormone and brain development: translating molecular mechanisms to population risk. Thyroid. 2003; 13(11):1001-10. [DOI:10.1089/105072 503770867165] [PMID]

4. Delange F. Neonatal screening for congenital hypothyroidism: Results and perspectives. Hormones. 1997; 48(2):5161. [DOI:10.1159/000185485]

5. Deladoëy J, Bélanger N, Van Vliet G. Random variability in congenital hypothyroidism from thyroid dysgenesis over 16 years in Quebec. The Journal of Clinical Endocrinology \& Metabolism. 2007; 92(8):3158-61. [DOI:10.1210/jc.20070527] [PMID]

6. Medda E, Olivieri A, Stazi MA, Grandolfo ME, Fazzini C, Baserga $\mathrm{M}$, et al. Risk factors for congenital hypothyroidism: Results of a population case-control study (1997-2003). European Journal of Endocrinology. 2005; 153(6):765-73. [DOI:10.1530/eje.1.02048] [PMID]

7. Pearce MS, Korada M, Day J, Turner S, Allison D, Kibirige M, et al. Increasing incidence, but lack of seasonality, of elevated TSH levels, on newborn screening, in the North of England. Journal of Thyroid Research. 2010; 2010(101948):1-5. [DOI:10.4061/2010/101948] [PMID] [PMCID]

8. Hinton CF, Harris KB, Borgfeld L, Drummond-Borg M, Eaton $\mathrm{R}$, Lorey $\mathrm{F}$, et al. Trends in incidence rates of congenital hypothyroidism related to select demographic factors: Data from the United States, California, Massachusetts, New 
York, and Texas. Pediatrics. 2010; 125(Suppl. 2):S37-47. [DOI:10.1542/peds.2009-1975D] [PMID]

9. Mao HQ, Yang RL, Liu ZH. [Correlation of congenital hypothyroidism with birth weight and gestational age in newborn infants (Chinese). Journal of Zhejiang University. Medical Sciences. 2007; 36(4):378-81. [PMID]

10. Silva SA, Chagas AJ, Goulart EM, Silva GA, Marçal LV, Gomes $\mathrm{MN}$, et al. Screening for congenital hypothyroidism in extreme premature and/or very low birth weight newborns: The importance of a specific protocol. Journal of Pediatric Endocrinology and Metabolism. 2010; 23(1-2):45-52. [DOI:10.1515/JPEM.2010.23.1-2.45]

11. Bijarnia S, Wilcken B, Wiley VC. Newborn screening for congenital hypothyroidism in very-low-birth-weight babies: The need for a second test. Journal of Inherited Metabolic Disease 2011; 34(3):827-33. [DOI:10.1007/s10545-0119286-8] [PMID]

12. Waller DK, Anderson JL, Lorey F, Cunningham GC. Risk factors for congenital hypothyroidism: An investigation of infant's birth weight, ethnicity, and gender in California, 1990-1998. Teratology. 2000; 62(1):36-41. [DOI:10.1002/1096-9926(200007)62:13.0.CO;2-W]

13. Thalhammer O. Screening for congenital hypothyroidism in Austria (author's transl). Klinische Padiatrie. 1981; 193(5):375-7. [DOI:10.1055/s-2008-1034501] [PMID]

14. Meberg A, Marstein S. Smoking during pregnancy-effects on the fetal thyroid function. Acta Paediatrica. 1986; 75(5):762-6. [DOI:10.1111/j.1651-2227.1986.tb10287.x]

15. Virtanen $M$, Mäenpää J, Pikkarainen J, Pitkänen L, Perheentupa J. Aetiology of congenital hypothyroidism in Finland. Acta Paediatrica. 1989; 78(1):67-73. [DOI:10.1111/j.1651-2227.1989.tb10889.x]

16. Kaiserman I, Siebner R, Kletter G, Sack J. A ten-year temporal analysis of primary congenital hypothyroidism in Israel. Early Human Development 1991; 26(3):193-201. [DOI:10.1016/0378-3782(91)90159-Z]

17. Lorey FW, Cunningham GC. Birth prevalence of primary congenital hypothyroidism by sex and ethnicity. Human Biology. 1992; 64(4):531-8. [PMID]

18. Sorcini M, Fazzini C, Olivieri A, Grandolfo M, Medda E, Stazi $M$, et al. [Neonatal screening in congenital hypothyroidism in Italy. The national registry (Italian)]. Annali Dell'Istituto Superiore Di Sanita. 1994; 30(3):275-87. [PMID]

19. Dussault JH. Screening for congenital hypothyroidism. Clinical Obstetrics and Gynecology. 1997; 40(1):117-23. [DOI:10.1097/00003081-199703000-00012] [PMID]

20. Hall S, Hutchesson A, Kirk J. Congenital hypothyroidism, seasonality and consanguinity in the West Midlands, England. Acta Paediatrica. 1999; 88(2):212-5. [DOI:10.1111/j.1651-2227.1999.tb01084.x] [PMID]
21. Rocchi MB, Perlini C, Ciatti R, Burroni M. Is the birthdate a risk factor for congenital hypothyroidism? A statistical answer based on personal experience. Minerva Pediatrica. 2001; 53(6):531-6. [PMID]

22. Henry G, Sobki SH, Othman JM. Screening for congenital hypothyroidism. Saudi Medical Journal. 2002; 23(5):52935. [PMID]

23. Ordookhani A, Mirmiran PA, Hedayati M, Hajipour R, Azizi F. [Screening for congenital hypothyroidism in Tehran and Damavand: An interim report on descriptive and etiologic findings, 1998-2001 (Persian)]. Iranian Journal of Endocrinology and Metabolism. 2002; 4(3):153-60.

24. Büyükgebiz A. Congenital hypothyroidism clinical aspects and late consequences. Pediatric Endocrinology Reviews. 2003; 1:185-90. [PMID]

25. Ouhoummane N, Levallois P, Gingras S. Thyroid function of newborns and exposure to chlorine dioxide by-products. Archives of Environmental Health. 2004; 59(11):582-7. [DOI:10.1080/00039890409603437] [PMID]

26. Ordookhani A, Hedayati M, Mirmiran P, Ainy E, SabetSaeedy $\mathrm{H}$, Azizi F. [Etiologies of transient congenital hypothyroidism in Tehran and Damavand (Persian)]. Iranian Journal of Endocrinology and Metabolism. 2004; 6(2):10713.

27. Lian XL, Bai Y, Xun YH, Dai WX, Guo ZS. [Effects of maternal hyperthyroidism and antithyroid drug therapy on thyroid function of newborn infants (Chinese)]. Zhongguo yi xue ke xue yuan xue bao. 2005; 27(6):756-60. [PMID]

28. McElduff A, McElduff $P$, Wiley V, Wilcken B. Neonatal thyrotropin as measured in a congenital hypothyroidism screening program: Influence of the mode of delivery. The Journal of Clinical Endocrinology and Metabolism. 2005; 90(12):6361-3. [DOI:10.1210/jc.2005-0786] [PMID]

29. Ordookhani A, Pearce EN, Mirmiran P, Azizi F, Braverman LE. The effect of type of delivery and povidone-iodine application at delivery on cord dried-blood-specimen thyrotropin level and the rate of hyperthyrotropinemia in mature and normal-birth-weight neonates residing in an iodine-replete area: Report of Tehran Province, 1998-2005. Thyroid. 2007; 17(11):1097-102. [DOI:10.1089/thy.2007.0058] [PMID]

30. Hashemipour M, Amini M, Kelishadi R, Hovsepian $S$, Haghighi $S$, Hosseini $M$, et al. Seasonal variation in the incidence of congenital hypothyroidism in Isfahan, Iran. Saudi Medical Journal. 2007; 28(10):1582-6. [PMID]

31. Gu YH, Kato T, Harada S, Inomata H, Saito T, Aoki K. Seasonality in the incidence of congenital hypothyroidism in Japan: Gender-specific patterns and correlation with temperature. Thyroid. 2007; 17(9):869-74. [DOI:10.1089/ thy.2006.0317] [PMID]

32. Hashemipour M, Amini M, Talaie M, Kelishadi R, Hovespian $\mathrm{S}$, Iranpour $\mathrm{R}$, et al. Parental consanguinity among parents of neonates with congenital hypothyroidism in Isfahan. 
Eastern Mediterranean Health Journal. 2007; 13(3):56774.

33. Olivieri A, Medda E, De Angelis S, Valensise H, De Felice M, Fazzini $\mathrm{C}$, et al. High risk of congenital hypothyroidism in multiple pregnancies. Journal of Clinical Endocrinology \& Metabolism. 2007; 92(8):3141-7. [DOI:10.1210/jc.20070238] [PMID]

34. Rowland K, Crotteau C, Kovach F, Hoekzema G. Clinical inquiries. What are the causes of elevated TSH in a newborn? The Journal of Family Practice. 2008; 57(3):185-7. [PMID]

35. Eftekhari N, Gh A, Khaksari M, Salari Z. [The prevalence rate of congenital hypothyroidism in Kerman/Iran in 2005-2007 (Persian)]. Journal of Kerman University of Medical Sciences. 2015; 15(3):243-50.

36. Sepandi M, Holakoei Naeini K, Yarahmadi S, Haghtdust A, Nedjat S, Taghdir M. [Risk factors for congenital hypothyroidism in Fars Province, Iran, 2003-2006 (Persian)]. Journal of School of Public Health and Institute of Public Health Research. 2009; 7(1):35-45.

37. Cranston MM, Ryan MA, Smith TC, Sevick CJ, Brodine SK. Hypothyroidism among military infants born in countries of varied iodine nutrition status. BMC Endocrine Disorders. 2010; 10:2. [DOI:10.1186/1472-6823-10-2] [PMID] [PM$\mathrm{CID}]$

38. Hashemipour M, Nasri P, Hovsepian S, Hadian R, Heidari $\mathrm{K}$, Attar HM, et al. Urine and milk iodine concentrations in healthy and congenitally hypothyroid neonates and their mothers. Endokrynologia Polska. 2010; 61(4):371-6. [PMID]

39. Aminzadeh M, Chomeili B, Riahi K, Dehdashtian M, Cheraghian $B$, Valavi E. Effect of temperature changes on the occurrence of congenital hypothyroidism. Journal of Medical Screening. 2010; 17(3):121-4. [DOI:10.1258/ jms.2010.010026] [PMID]

40. Hashemipour M, Hasani N, Amini M, Heidari K, Sajadi A, Dastanpour $M$, et al. Thyroid function abnormalities among first-degree relatives of Iranian congenital hypothyroidism neonates. Pediatrics international. 2010; 52(3):467-71. [DOI:10.1111/j.1442-200X.2009.03016.x] [PMID]

41. Safar alizade F, Sadify R, Parto Azam H. [Prevalence of congenital hypothyroidism and its relation with some risk factors in Khoy health service centers (Persian)]. Journal of Urmia Nursing and Midwifery Faculty. 2010; 8(1):35-9.

42. Stoppa-Vaucher S, Van Vliet G, Deladoëy J. Variation by ethnicity in the prevalence of congenital hypothyroidism due to thyroid dysgenesis. Thyroid. 2011; 21(1):13-8. [DOI:10.1089/thy.2010.0205] [PMID] [PMCID]

43. Hashemipour M, Abari SS, Mostofizadeh N, HaghjooyJavanmard S, Esmail N, Hovsepian S, et al. The role of maternal thyroid stimulating hormone receptor blocking antibodies in the etiology of congenital hypothyroidism in Isfahan, Iran. International Journal of Preventive Medicine. 2012; 3(2):128-33. [PMID] [PMCID]
44. Zeinalzadeh AH, Talebi M. Neonatal screening for congenital hypothyroidism in East Azerbaijan, Iran: The first report. Journal of Medical Screening. 2012; 19(3):123-6. [DOI:10.1258/jms.2012.012024] [PMID]

45. Ooki S. Congenital hypothyroidism after assisted reproductive technology in Japan: Comparison between multiples and singletons, 2005-2009. International Journal of Pediatric Endocrinology. 2013; 2013:5. [DOI:10.1186/1687-98562013-5] [PMID] [PMCID]

46. Abdelmoktader AM. Risk factors for congenital hypothyroidism in Egypt: Results of a population case-control study (2003-2010). Annals of Saudi Medicine. 2013; 33(3):273-6. [DOI:10.5144/0256-4947.2013.273] [PMID] [PMCID]

47. Rezaeian S, Poorolajal J, Moghimbegi A, Esmailnasab N. Risk factors of congenital hypothyroidism using propensity score: A matched case-control study. Journal of Research in Health Sciences. 2013; 13(2):151-6.

48. Ng SM, Wong SC, Paize F, Chakkarapani E, Newland P, Isherwood $D$, et al. Multivariate analyses of factors that affect neonatal screening thyroid stimulating hormone. Journal of Pediatric Endocrinology \& Metabolism. 2011; 24(910):727-32. [DOI:10.1515/JPEM.2011.234] [PMID]

49. Dalili S, Rezvany SM, Dadashi A, Medghalchi A, Mohammadi $\mathrm{H}$, Dalili $\mathrm{H}$, et al. Congenital hypothyroidism: A review of the risk factors. Acta Medica Iranica. 2012; 50(11):735-9.

50. Kırmızıbekmez H, Güven A, Yıldız M, Cebeci AN, Dursun F. Developmental defects of the thyroid gland: Relationship with advanced maternal age. Journal of Clinical Research in Pediatric Endocrinology. 2012; 4(2):72-5. [DOI:10.4274/ Jcrpe.560] [PMID] [PMCID]

51. Rabbiosi S, Vigone MC, Cortinovis F, Zamproni I, Fugazzola L, Persani L, et al. Congenital hypothyroidism with eutopic thyroid gland: Analysis of clinical and biochemical features at diagnosis and after re-evaluation. The Journal of Clinical Endocrinology and Metabolism. 2013; 98(4):1395-402. [DOI:10.1210/jc.2012-3174] [PMID]

52. Esmailnasab N, Moasses G, Afkhamzadeh A. [Investigation of the risk factors for congenital hypothyroidism in the newborns in Kurdistan Province (Persian)]. Scientific Journal of Kurdistan University of Medical Sciences. 2012; 17(4):103-8.

53. Rezaeian S, Moghimbeigi A, Esmailnasab N. Gender differences in risk factors of congenital hypothyroidism: An interaction hypothesis examination. International Journal of Endocrinology and Metabolism. 2014; 12(2):e13946. [DOI:10.5812/ijem.13946] [PMID] [PMCID]

54. Dorreh F, Chaijan PY, Javaheri J, Zeinalzadeh AH. Epidemiology of congenital hypothyroidism in Markazi Province, Iran. Journal of Clinical Research in Pediatric Endocrinology. 2014; 6(2):105-10. [DOI:10.4274/jcrpe.1287] [PMID] [PMCID]

55. Uenaka M, Tanimura K, Tairaku S, Morioka I, Ebina Y, Yamada H. Risk factors for neonatal thyroid dysfunction in preg- 
nancies complicated by Graves' disease. European Journal of Obstetrics, Gynecology, and Reproductive Biology. 2014; 177:89-93. [DOI:10.1016/j.ejogrb.2014.03.007] [PMID]

56. Fan X, Chen S, Qian J, Sooranna S, Luo J, Li C, et al. Incidence and interrelated factors in patients with congenital hypothyroidism as detected by newborn screening in Guangxi, China. Global Pediatric Health. 2015; 2:2333794X14567193. [DOI:10.1177/2333794X14567193] [PMID] [PMCID]

57. Satoh M, Aso K, Katagiri Y. Thyroid dysfunction in neonates born to mothers who have undergone hysterosalpingography involving an oil-soluble iodinated contrast medium. Hormone Research in Paediatrics. 2015; 84(6):370-5. [DOI:10.1159/000439381] [PMID]

58. Mehrnejat N, Yazdanpanah H, Nobari RF, Hashemipour $M$, Maracy $M$, Moafi $M$, et al. Spatial analysis of neonatal congenital hypothyroidism and nitrate as an environmental pollutant in Isfahan Province during 2010-2013. International Journal of Preventive Medicine. 2015; 6. [DOI:10.4103/2008-7802.162952] [PMID] [PMCID]

59. Zhou J, Luo J, Zhao H, Wang J, Lin F, Zhang H, et al. [Risk factors of 125 cases of neonatal congenital hypothyroidism during perinatal period (Chinese)]. Zhonghua liu xing bing xue za zhi Zhonghua liuxingbingxue zazhi. 2015; 36(7):74751. [PMID]

60. Trumpff C, Vandevijvere S, Moreno-Reyes R, Vanderpas J, Tafforeau J, Van Oyen $\mathrm{H}$, et al. Neonatal thyroid-stimulating hormone level is influenced by neonatal, maternal, and pregnancy factors. Nutrition Research. 2015; 35(11):97581. [DOI:10.1016/j.nutres.2015.09.002] [PMID]

61. Dayal D, Prasad R. Congenital hypothyroidism: Current perspectives. Research and Reports in Endocrine Disorders. 2015; 5(5):91-102. [DOI:10.2147/RRED.S56402]

62. Keshavarzian E, Valipoor AA, Maracy MR. The incidence of congenital hypothyroidism and its determinants from 2012 to 2014 in Shadegan, Iran: A case-control study. Epidemiology and Health 2016; 38:e2016021. [DOI:10.4178/epih. e2016021] [PMID] [PMCID]

63. Aguiar L, Garb J, Reiter E, Visintainer P, Singh R, Allen H, et al. Can one predict resolution of neonatal hyperthyrotropinemia? The Journal of Pediatrics. 2016; 174:71-7.e1. [DOI:10.1016/j.jpeds.2016.04.011] [PMID]

64. Blasig S, Kühnen P, Schuette A, Blankenstein O, Mittag J, Schomburg L. Positive correlation of thyroid hormones and serum copper in children with congenital hypothyroidism. Journal of Trace Elements in Medicine and Biology. 2016; 37:90-5. [DOI:10.1016/j.jtemb.2016.05.007] [PMID]

65. Yang HH, Qiu L, Zhao JQ, Yang N, Gong LF, Kong YY. [Epidemiologic characteristics and risk factors for congenital hypothyroidism from 1989 to 2014 in Beijing (Chinese)]. Zhonghua yu fang yi xue za zhi. 2016; 50(8):728-32. [DOI:10.3760/cma.j.issn.0253-9624.2016.08.011]

66. Anastasovska V, Kocova M. Ethnicity and incidence of congenital hypothyroidism in the capital of Macedonia.
Journal of Pediatric Endocrinology and Metabolism. 2017; 30(4):405-9. [DOI:10.1515/jpem-2016-0178]

67. Khanjani N, Ahmadzadeh A, Bakhtiari B, Madadizadeh F. The role of season and climate in the incidence of congenital hypothyroidism in Kerman province, Southeastern Iran. Journal of Pediatric Endocrinology and Metabolism. 2017; 30(2):149-57. [DOI:10.1515/jpem-2016-0178] [PMID]

68. Castanet M, Polak M, Bonaiti-Pellie C, Lyonnet S, Czernichow $P$, Leger J. Nineteen years of national screening for congenital hypothyroidism: Familial cases with thyroid dysgenesis sug-gest the involvement of genetic factors. The Journal of Clinical Endocrinology \& Metabolism. 2001; 86(5):2009-14. [DOI:10.1210/jcem.86.5.7501] [PMID]

69. Rosenthal M, Addison GM, Price DA. Congenital hypothyroidism: Increased incidence in Asian families. Archives of Disease in Childhood. 1988; 63(7):790-3. [DOI:10.1136/ adc.63.7.790] [PMID] [PMCID] 